ÉGYPTE monde arabe

\section{Égypte/Monde arabe}

$34 \mid 1998$

Droits d'Égypte : histoire et sociologie

\title{
Repères pour une praxéologie de l'activité juridique
}

Le traitement judiciaire de la moralité à partir d'un exemple égyptien

Baudouin Dupret

\section{OpenEdition}

Journals

Édition électronique

URL : https://journals.openedition.org/ema/1507

DOI : 10.4000/ema. 1507

ISSN : 2090-7273

Éditeur

CEDEJ - Centre d'études et de documentation économiques juridiques et sociales

Édition imprimée

Date de publication : 31 décembre 1998

Pagination : 115-140

ISSN : 1110-5097

Référence électronique

Baudouin Dupret, « Repères pour une praxéologie de l'activité juridique », Égypte/Monde arabe [En ligne], 34 | 1998, mis en ligne le 08 juillet 2008, consulté le 07 juillet 2022. URL : http://

journals.openedition.org/ema/1507 ; DOI : https://doi.org/10.4000/ema.1507

Ce document a été généré automatiquement le 7 juillet 2022

Tous droits réservés 


\section{Repères pour une praxéologie de l'activité juridique}

Le traitement judiciaire de la moralité à partir d'un exemple égyptien

\section{Baudouin Dupret}

1 L'espace public égyptien semble très largement déterminé par la question de la moralité publique et de sa définition. On peut même penser que le propre d'un répertoire normatif comme celui de la sharî'a n'est pas tant de renvoyer à un système juridique particulier que d'imposer, au moins négativement, la moralisation du débat (Ferrié, 1997a et b). J'entends par négativement le fait que ce répertoire n'impose pas de se conformer à un ensemble défini de normes précises, mais contraint toute évaluation morale à passer par un mécanisme de justification par rapport à ce répertoire normatif. Se pose dès lors la question des modes constitutifs de cette moralité, de sa traduction juridique et de l'interrelation qu'on peut y observer des sens professionnel (ou judiciaire) et profane (ou ordinaire) du droit. Cet article tente de décrypter certains des mécanismes qui permettent d'expliquer que la moralité se construise de manière interactionnelle.

2 La psychologie cognitive étudie le processus de catégorisation, tandis que la théorie juridique s'intéresse à l'opération de qualification. L'usage de deux termes différents (catégorisation et qualification) a tendance à établir une dichotomie entre les opérations cognitives propres au sens commun et les procédures techniques liées au travail professionnel. Pourtant, les sens ordinaire et judiciaire ne peuvent être complètement dissociés, de la même façon que le sociologue, pour reprendre le langage ethnométhodologique, ne peut prétendre à une position en «surplomb» de la réalité sociale, du sens commun la décrivant et des pratiques l'habitant, toutes choses qu'il regarderait de manière « ironique ». Plutôt donc que de considérer les deux opérations séparément, je propose d'utiliser un même outil analytique : la typification. Dans cette perspective, la typification ne consiste pas dans l'attribution à une situation des qualités lui permettant de relever d'une catégorie (opération prédicative), mais dans l'opération d'attribution elle-même, dans la formule des opérations possibles face à une situation donnée. Cette approche par la typification permet à la fois de réduire 
l'opposition des sens ordinaire et judiciaire et de prendre la mesure de ce que la pragmatique propre aux différentes activités ordinaires et professionnelles implique comme différenciation dans la construction du sens. En d'autres mots, il devrait être possible de ne plus opposer les sens ordinaire et judiciaire sur le plan sémantique tout en les distinguant sur le plan pragmatique.

3 Je ne reviendrai pas sur les développements de la psychologie cognitive en matière de catégorisation et de la théorie juridique en matière de qualification, auxquels je m'attache plus longuement dans un autre travail (Dupret, 1998). Je m'attacherai ici à démontrer qu'il n'y a pas lieu d'établir une dichotomie entre sens commun du droit et savoir technique juridique, de la même façon qu'il ne faudrait pas complètement opposer sens ordinaire et savoir sociologique. La notion de typification servira d'outil. On l'envisagera d'abord dans une perspective sémiotique et on proposera par après de lui apporter des ajustements tirés de la socio-phénoménologie. Je chercherai alors à montrer que les sens ordinaire et judiciaire du droit et de la justice sont constitués de manière croisée, confondue et doublement réflexive et que leur différenciation tient davantage à la question de leur pragmatique qu'à celle de leur sémantique. Une affaire de viol collectif survenue en janvier 1985, au Caire, sert de support à l'analyse.

\section{Sens commun et savoir juridique}

4 Je voudrais d'abord faire quelques remarques sur la position du professionnel du droit par rapport à l'objet dont il est saisi. En un mot, je prétends qu'à l'image du sociologue dans son rapport au savoir social, le juriste n'occupe pas une position totalement distincte et distanciée par rapport aux intrigues sociales qu'il doit investir de son savoir et au savoir social qui porte déjà sur ces intrigues.

L'acte de connaissance est social. Comme le dit Schütz :

« Toute notre connaissance du monde, qu'elle s'exprime dans la pensée courante ou dans la pensée scientifique, comprend des constructions, par exemple, un ensemble d'abstractions, de généralisations, de formalisations et d'idéalisations spécifiques au niveau spécifique d'organisation de la pensée où l'on se trouve. À strictement parler, il n'y a pas de choses, telles que des faits purs et simples. Tous les faits sont d'emblée sélectionnés dans un contexte universel par les activités de notre esprit. Ils sont donc toujours des faits interprétés ou des faits considérés comme détachés de leur contexte par une abstraction artificielle ou alors des faits considérés dans leur organisation particulière. Dans les deux cas, ils portent en eux leur horizon d'interprétation interne et externe. Cela ne signifie pas que, dans la vie quotidienne ou dans la science, nous soyons incapables de saisir la réalité du monde. Cela signifie simplement que nous n'en saisissons que certains aspects, notamment ceux qui sont pertinents pour nous, soit pour gérer notre propre vie, soit du point de vue du corpus de règles de procédure de pensée admises telles quelles, appelé méthode scientifique. » (Schütz, 1987, p. 9)

6 On constate ainsi que « la pertinence n'est pas intérieure à la nature en tant que telle », mais qu'elle constitue «le résultat de l'activité sélective et interprétative de l'homme dans la nature ou dans la nature qu'il observe » (id., p. 10). Ce qui différencie les deux perspectives professionnelle et profane est, en fin de compte, une question de degré. Pour reprendre Schütz :

«Les objets de pensée, construits par les chercheurs en sciences sociales, se fondent sur les objets de pensée construits par la pensée courante de l'homme menant sa vie quotidienne parmi ses semblables et s'y référant. Ainsi, les constructions 
utilisées par le chercheur en sciences sociales sont, pour ainsi dire, des constructions au second degré, notamment des constructions de constructions édifiées par les acteurs sur la scène sociale dont l'homme de science observe le comportement et essaie de l'expliquer tout en respectant les règles de procédure de sa science. » (id., p. 11)

7 Professionnels et profanes construisent un monde de typicalités à partir de leur situation biographique. "Dans les deux cas, ces constructions ont une place particulière le long de la chaîne des motifs s'originant dans la hiérarchie biographiquement déterminée des plans du constructeur » (id, p. 48). Le savant prétend se distinguer du profane par sa position désintéressée - «il n'a pas d'"ici" à l'intérieur du monde social» (ibid.) - et la maîtrise d'un corpus de savoir distinct et structuré différemment, mais, au même titre que le profane, il est engagé socialement dans l'action de production du savoir.

8 L'insertion dans des univers distincts justifie l'adoption de méthodes distinctes, non la détention, par le professionnel, d'un monopole du savoir et de l'objectivation du social. Telle est la position de l'ethnométhodologie pour laquelle «la coupure épistémologique entre connaissance pratique et connaissance savante n'est pas de même nature que celle qui est habituellement admise par les sociologues"(Coulon, 1987, p.50). Le professionnel n'occupe pas de position de surplomb lui permettant de décrypter les règles auxquelles l'individu, considéré comme un «idiot culturel», ne ferait que se conformer. L'un et l'autre font de la "sociologie », utilisent des "ethnométhodes ", typifient et catégorisent le monde, en un mot, produisent une visibilité du social permettant d'agir sur lui. Dans cette démarche, l'un et l'autre adoptent ce que Garfinkel a appelé, à la suite de Mannheim qui réservait toutefois l'expression à la seule connaissance savante, une méthode documentaire d'interprétation. Ils recherchent le modèle (un pattern, disent les ethnométhodologues) homologue sous-jacent qui est à la fois désigné par l'occurrence et qui en même temps la désigne. «Ce modèle sous-jacent est non seulement dérivé des preuves documentaires individuelles, mais les preuves documentaires individuelles à leur tour sont interprétées sur la base de ce qui est "connu" de ce modèle sous-jacent. Chacun est utilisé pour élaborer l'autre » (Garfinkel, 1967, p. 78)1. Dans cette démarche toujours, profane et professionnel procèdent de manière indexicale et réflexive. L'indexicalité traduit le fait que, pour chaque individu, la signification du langage qu'il utilise dépend du contexte dans lequel ce langage apparaît, donc de ses conditions d'usage et d'énonciation. Ceci vaut aussi des gestes, des énoncés, des actions, etc., toutes choses entourées d'une " frange d'incomplétude » tant qu'elles ne se produisent pas et dont l'accomplissement même annonce un « horizon d'incomplétude ». C'est ce que Cicourel a appelé le " caractère rétrospectifprospectif des événements " (Cicourel, 1979, p. 69-71). La réflexivité traduit, quant à elle, cette propriété qu'ont les activités de présupposer en même temps que de rendre observable la même chose. Elle constitue cet acte performatif qui, dans la description du social qu'il opère, produit ce social au travers de "l'acquiescement motivé des personnes qui ont déjà ces attentes" (Garfinkel, 1967, p. 55). "La réflexivité désigne l'équivalence entre décrire et produire une interaction, entre la compréhension et l'expression de cette compréhension » (Coulon, 1987, p. 38). C'est la pratique qui fait la règle, l'interaction qui dit le code, et non la règle qui conditionne la pratique ou le code qui détermine l'interaction : seule une actualisation constante permet à la règle ou au code d'exister. Ce qui dès lors distingue encore le profane du professionnel tient donc 
davantage au contexte de leurs performances respectives, à leur appartenance à des « communautés » différentes engagées dans des « pragmatiques » différentes.

Pour reprendre l'expression de Heidegger, le sociologue est projeté au cœur du phénomène qu'il se propose d'analyser. Cela signifie par conséquent que

«toute investigation est inévitablement entrelacée, et cela dès le départ, avec le phénomène qu'elle étudie ; ensuite, que le sociologue - et en cela il est logé à la même enseigne que tout autre membre de la société - doit réaliser ses enquêtes effectives sous les auspices des attentes partagées d'arrière-plan et de la compréhension de sens commun, qui sont les siennes en tant que membre de la société. L'idée est donc que pour être en mesure de faire quelque proposition que ce soit sur les faits observés dans des situations sociales, le sociologue doit nécessairement mobiliser une part de la compréhension de sens commun " (Sharrock, Watson, 1990, p. 229).

Bien entendu, cela ne signifie nullement que le sociologue fasse siennes les exigences et prétentions des acteurs (id., p. 230), mais simplement qu'il s'intéresse à l'ordre social tel que tout un chacun - professionnel et profane - le découvre, plutôt qu'à l'ordre social tel que produit par une quelconque méthodologie professionnelle, et qu'il cherche systématiquement la source de cet ordre dans les activités manifestant des régularités bien connues (id., p. 236-237). Ainsi, les ethnométhodologues choisissent de regarder

«comment les activités s'organisent elles-mêmes, comment ceux qui se meuvent dans un cadre social donné peuvent organiser leurs activités de telle façon qu'elles reproduisent les régularités qu'on s'attend à trouver dans de telles situations et qu'elles incarnent des façons de faire qui peuvent être reconnues comme normales par ceux qui occupent ces mêmes lieux - car les régularités que ces activités manifestent sont après tout familières, reconnaissables et bien connues pour quiconque fréquente ces lieux » (id., p. 237).

11 D'où la notion de "contextes qui s'expliquent eux-mêmes", qui permet de «caractériser certaines dimensions selon lesquelles une scène locale d'activité s'organise elle-même de façon à manifester l'allure familière du type d'activité routinisée auquel on a coutume d'assister en cet endroit » (ibid.). Sharrock et Watson donnent ainsi l'exemple des réponses du juge aux remarques des accusés, réponses qui constituent pour cet accusé autant d'instructions lui permettant de déterminer comment s'exprimer au tribunal, mais aussi réponses pouvant instruire les spectateurs sur la manière dont les accusés doivent généralement se conduire - «ce qui peut considérablement les aider quand leur tour sera arrivé à se comporter eux-mêmes de façon standardisée, et à se défendre comme on le fait généralement » (id., p. 238). Le contexte est alors qualifié d'«auto-explicatif» (self-explicating) dans la mesure où il indique «la façon dont l'organisation propre du cadre d'un tribunal apprend à des participants non informés comment finalement "ce tribunal" se comporte » (ibid). II est aussi l'occasion d'émergence de pertinences, selon la formule de Schütz :

«L'expression d'un second énoncé manifeste le sens que le second locuteur donne aux énoncés du premier, et celui-ci peut utiliser les exigences normatives projetées sur le second par sa propre expression pour comprendre et évaluer celle de son destinataire. » (id., p. 240)

12 Les catégorisations spontanées des relations sexuelles que des magistrats égyptiens m'ont proposées peuvent servir d'appui à cette affirmation qu'il n'y a pas de déconnexion radicale entre le juriste et le profane et, partant, entre les sens ordinaire et judiciaire du droit et de la justice. Pour les différents magistrats, tous de sexe masculin, avec qui je me suis entretenu, la catégorie « relations sexuelles licites », c'est- 
à-dire des relations unissant, dans le cadre d'un mariage légal, un homme et une femme majeurs, constitue la référence de base pour l'évaluation de tout acte sexuel. La contrainte n'apparaît pas prépondérante, de sorte que l'idée de viol est exclue à l'intérieur d'un couple légalement marié. Comme le paradigme "relations sexuelles licites » implique les relations d'un homme et d'une femme dans le cadre du mariage par toute intromission d'un organe de l'homme ou d'un objet qu'il tient dans le sexe de la femme, le viol, qui est l'accomplissement de relations sexuelles illicites par contrainte, suppose nécessairement un homme et une femme et la relation décrite plus haut. Cette catégorisation du viol exclut dès lors toute inversion de rôle des protagonistes (une femme ne peut pas violer un homme), toute relation qui ne serait pas strictement celle décrite plus haut (comme la sodomie) et toute situation homosexuelle (un homme ne peut pas violer un homme).

D'un autre côté, à parcourir le traitement profane et médiatique des circonstances et du jugement de l'affaire de "la jeune fille de Ma'âdî", ce procès des six jeunes gens, de 18 à 30 ans, accusés d'avoir enlevé dans le quartier de Ma'âdî, au Caire, d'avoir séquestré et dépouillé une jeune femme et son fiancé et d'avoir violé la première, on ne manque pas de tomber sur une série d'images que l'on qualifierait volontiers, dans une perspective de psychologie cognitive, de paradigmatiques de la pudeur féminine, de la sexualité, de son contrôle et de la répression de la transgression. Qu'il s'agisse de l'appellation générale de cette affaire de mœurs, qadiyyat fatât al-Ma'âdî (l'affaire de la jeune fille de Ma'âdî), ou de certains titres et sous-titres de la presse, l'action semble toujours catégorisée par son rattachement à un archétype. Il s'agit ici de la catégorie de " crime répugnant " (jarîma bashi'a). Celle-ci semble regrouper, dans la presse, tous les types d'affaires de mœurs, de la pornographie à la prostitution et au viol. La présentation de l'affaire de Ma'âdî la fait tomber sous l'évidence de cette appartenance catégoriale. On trouve ainsi, en titre, cette déclaration de la jeune fille : «J'ai fait face à des monstres qui m'ont déchiré la peau de leurs crocs " (wâjahtu wuhûshan mazzaqû admiyatî bi-anyâbihim). Le traitement médiatique d'une autre affaire de viol survenue en mars 1992, l'affaire de "la jeune fille de 'Ataba " (qadiyyat fatât 'Ataba), confirme ce fonctionnement paradigmatique. Il est ainsi question d'une jeune fille qui, un soir du " mois béni de Ramadan ", a fait l'objet d'une " agression répugnante ». On peut noter que, parmi les éléments constitutifs du paradigme "crime répugnant ", la question de la virginité occupe une place prépondérante. Le fait par exemple qu'aux dires du médecin légiste, la jeune femme de Ma'âdî soit demeurée vierge, en dépit de l'agression sexuelle collective dont elle a fait l'objet, constitue de toute évidence un problème, dans l'opération de catégorisation des journalistes, que seule une explication médicale circonstanciée a pu compenser.

Ce type de catégorisation n'est cependant pas le fait exclusif des journalistes. On perçoit l'entrecroisement des sens professionnel et ordinaire du droit et de la justice dans des textes divers mettant en parallèle les dispositions du droit positif et les règles attribuées à la sharî'a islamique en matière de viol. J'ai déjà eu l'occasion de montrer que, s'agissant de la sharîa, les magistrats égyptiens semblent tributaires d'une « information sociale » qui conditionne leur propre perception métaphysique de la religion, de sa place dans la société que le droit dont ils sont porteurs prétend organiser et, dès lors, du champ des "possibles » et « impossibles » dans lequel inexorablement ils sont situés. Le professionnel du droit se trouve ainsi au centre d'une tension entre dynamiques normatives divergentes : celle de la science du droit positif et celle de la 
logique de la morale prédominante, elle-même traversée de courants multiples correspondant à la multiplicité des standards de vie (Dupret, 1995). Ainsi, on mentionnera la définition jurisprudentielle qui est donnée aux parties sexuées :

«\$84 - Applications jurisprudentielles du critère de "parties sexuelles" (mi'yâr al-'awra) : la jurisprudence considère les actes suivants comme une atteinte à la pudeur de la victime du fait du contact avec les parties sexuelles de son corps: l'accusé étend la victime (femme) au sol et rompt son hymen de son doigt; pincer une femme au derrière; l'accusé étreint le sein de la victime (femme); pincer la victime (femme) à la cuisse ; l'accusé étend la victime (femme) au sol sur le dos, se couche sur elle et met son doigt dans son derrière ; étreindre la victime (femme); l'accusé serre la victime (femme) entre ses mains ; l'accusé déchire le pantalon de la victime (femme) et s'introduit [...] dans son vagin; l'accusé s'étend sur la victime (femme) après l'avoir mise au sol; arracher les vêtements d'une partie du corps de la victime (femme) recouvrant des parties sexuelles et les exhiber pour les regarder; l'accusé met sa main sur le vagin de la première victime (femme), palpe le ventre et les seins de la seconde et saisit le genou et le ventre de la troisième. En application de ce critère, la jurisprudence ne considère pas le fait d'embrasser une jeune fille sur les joues ou d'embrasser un jeune homme sur la nuque et de le mordre à l'endroit du baiser comme une atteinte à la pudeur de tous deux. » (Najî́b Hasan, «Droit de la protection de la pudeur » (en arabe), Majallat al-qânûn wa liqtisâd - huqûq al-insân).

Dans cette perspective, on relèvera également qu'un professeur de droit traitant du viol du double point de vue de la sharî'a et du droit positif, introduit et conclut son article par l'apologie des vertus et mérites du mariage et par la nécessité de renforcer la sanction de l'atteinte portée à ce seul cadre légal pour les relations sexuelles (Ahmad Kâmil Salâma, professeur à la faculté de droit du Caire, al-Gumhûriyya, mars 1985). Il ressort de cette présentation que la moralité sexuelle s'articule très clairement, dans sa double dimension professionnelle et profane, autour de la notion d'exclusivité maritale des relations sexuelles, le viol ne constituant de la sorte que la contradiction de cet idéal aggravé par l'usage de la contrainte.

L'usage constant, dans l'affaire de la jeune fille de Ma'âdî, de la notion de virginité, que ce soit dans les documents légaux ou dans les articles de presse, atteste aussi de l'importance prise par un concept qui n'est pas strictement juridique dans la narration juridique ou journalistique d'une atteinte à la pudeur ou d'un viol. La préservation de l'hymen de la jeune fille constituait certes une question à résoudre pour attester du viol. Il n'en reste pas moins que l'insistance sur sa virginité ne peut être limitée à une simple considération technique. On verra plus loin à quel point ce type de considérations organisent la catégorisation des protagonistes et les droits et devoirs qui s'y attachent. On touche ici à l'organisation linguistique, contextuelle et indexicale du sens. Cette insistance n'est pas non plus le fait exclusif de la presse: Parquet et juge s'appuient également sur une organisation narrative dichotomique opposant victime et agresseurs, « la jeune fille vierge » et « les six loups».

\section{Un outil analytique : la typification}

17 Comme je l'ai déjà dit en introduction, on devrait converger, si l'on accepte la réduction de la fracture entre profanes et professionnels que la distinction des opérations de catégorisation et de qualification semblait avoir introduite, sur la nécessité de se doter d'un outil analytique permettant d'analyser d'un même 
mouvement l'appréhension du donné juridique. Je propose de trouver dans la notion procédurale de typification l'outil en question.

Bernard Jackson propose un modèle d'analyse du droit en termes de typifications narratives qui se fonde sur l'approche sémiotique greimassienne (Jackson, 1995). Jackson emprunte à Fletcher (1978) la notion d'image collective. Partant de la question du rapprochement de situations factuelles avec une image collective (par exemple, celle d' "agir comme un voleur»), il développe l'idée d'un paradigme à distance plus ou moins grande duquel se situent les occurrences qui surviennent et qui sont évaluées par les différents acteurs sociaux. L'image collective est ainsi à la fois la description d'une action typique et l'évaluation sociale de sa performance. Le rapprochement d'avec l'image collective constitue de la sorte un transfert de modalité. C'est ce que Bernard Jackson qualifie de "typification narrative de l'action ». On peut en relever trois propriétés : (a) elle ne génère pas de jugements démontrables de ce qui relève ou non de l'image collective (ce n'est pas une définition en termes de conditions nécessaires et suffisantes), mais elle produit des jugements de similarité relative ; (b) pareille typification n'est pas une description neutre, mais est chargée d'une forme d'évaluation; (c) certaines typifications sont propres à certains groupes sémiotiques que des systèmes de significations propres distinguent les uns des autres (bien qu'ils puissent partiellement se superposer). Tout ce savoir social est intériorisé par les membres du groupe concerné et est mobilisé de manière plus ou moins consciente, ce qui signifie que certaines typifications narratives sont intériorisées au sein d'un groupe particulier. Les typifications narratives ne fonctionnent pas mécaniquement, mais, au contraire, de manière négociée et interactionniste.

19 Le droit, qu'il s'agisse des règles, des décisions ou autres réquisitoires et plaidoiries, peut être envisagé comme l'ensemble des modèles narratifs socialement construits de l'expérience humaine (Jackson, 1988, p.101), mis en œuvre dans un cadre institutionnel. La décision judiciaire, pour ne prendre qu'elle, devient alors une procédure de comparaison, plus évaluative que constative, d'unités narratives concurrentes, celle(s) des faits et celle(s) de la règle. De manière générale, la pratique professionnelle du droit est une forme (ou, plus exactement, un ensemble de formes) de comportement humain productrice de sens. Ce sens n'est pas propre à la pratique juridique, il lui est imputé par ceux qui y participent. C'est en quelque sorte le sens commun de ceux qui sont engagés dans une certaine pratique. Notons déjà que le sens ne doit pas tant être entendu d'un point de vue sémantique que pragmatique :

«En termes sémiotiques, le sens de la pratique professionnelle est autant une question des significations attribuées à sa pragmatique (comment les gens font usage de systèmes de signification) que de celles qui sont attribuées à sa sémantique (ce qu'ils disent au travers de l'usage de pareils systèmes). » (Jackson, 1994, p. 55)

L'insertion du sens dans le cadre social de sa construction - le sens est fonction du groupe sémiotique qui en fait usage, il est usage plus qu'essence, ressource plus que source - suppose qu'il fasse l'objet d'accumulations: l'individu, biographiquement, emmagasine des cadres de compréhension dans lesquels il tendra à inscrire ses nouvelles expériences. Au titre de ces cadres, on citera les différentes variantes de l'activité juridique.

21 On ne saurait reprocher à Bernard Jackson de sous-estimer la dimension pragmatique de l'activité juridique au profit de sa dimension sémantique. Il est toutefois possible d'adresser un certain nombre de critiques à la notion de typification telle qu'il la 
construit d'un point de vue sémiotique. Proche de la perspective propre à la psychologie cognitive et à l'image des théories classiques du concept (Cassirer, 1969 et 1972, cité par Quéré, 1994, p. 18), il accorde une préséance logique à la catégorie de substance, fait de la relation de subsomption d'un contenu à un autre une relation constitutive, attribue un caractère classificatoire à l'acte d'identification des objets et phénomènes (l'identité d'un objet est déterminée par la classe au travers de laquelle il est connu), réduit la conscience de la catégorie à la conscience d'une représentation (le raisonnement n'est alors qu'une aptitude à reproduire des contenus représentatifs), conçoit la construction des concepts comme un processus d'abstraction et de comparaison et considère les mots du langage comme des reproductions d'essences déterminées de la nature et du monde des représentations. Il faut pourtant remarquer que la possibilité même de décomposer un objet représenté en ses caractéristiques et de le rapporter à d'autres suppose déjà d'avoir son concept et que l'accent mis sur le fonctionnement par extension du concept se fait aux dépens de la compréhension qu'il permet (le concept permet d'imposer une forme aux impressions pour en faire des représentations). Il conviendrait sans doute d'adopter, au contraire, une perspective plus praxéologique: de même que le concept met en œuvre des procédures réglées d'analyse, de composition et de combinaison des objets (le concept est la règle ou la méthode de ces procédures), la catégorisation est un moment dans un processus de descente en singularité qui fonctionne selon un principe de sélection visant à détacher un moment caractéristique et à en faire le point sur lequel se dirige l'attention (Quéré, 1994, p. 20).

La phénoménologie husserlienne permet de voir que la catégorisation doit être appréhendée en deçà du jugement prédicatif (ce n'est pas la subsomption d'un objet sous une classe d'appartenance). Husserl parle de "préconnaissance typique de tout objet d'expérience singulier ». Repris en termes sociologiques par Schütz, cela signifie que nous saisissons d'emblée les objets, les événements, les personnes comme étant d'une certaine sorte, nous les appréhendons dans leurs propriétés typiques, c'est-à-dire selon des déterminations générales, liées au type, et non pas dans leurs particularités individuelles. La perspective ainsi ouverte permet d'étendre la valeur opératoire des catégories aux domaines de la construction intersubjective de l'objectivité et de l'organisation des activités pratiques. Si l'on fait de la catégorie une formule d'opérations possibles, cela entraîne qu'elle concerne

« non seulement les prédicats, les modes de prédication ou les cadres formels de la pensée, mais aussi les concepts des entités de base à l'aide desquelles nous individuons les phénomènes (personne, objet, événement, fait, action, passion, etc.) ainsi que les concepts qui présentent la propriété particulière d'être constitutifs de l'objectivité des phénomènes qu'ils décrivent (ils sont "autoréférentiels", dit Searle) et donc de participer, en tant que représentations ou croyances, schèmes ou méthodes, à l'institution de la réalité objective du monde social » (Quéré, 1994, p. 30).

Je propose de considérer la typification dans une perspective socio-phénoménologique et dans ses prolongements ethnométhodologiques. Je me permettrai, à cet effet, de reprendre le résumé qu'en fait Alain Coulon (1994).

«Le monde social de Schütz est celui de la vie quotidienne, vécue par des individus de sens commun, avec leurs pensées et leurs émotions de tous les jours, et qui ne portent pas a priori d'intérêt théorique à la constitution du monde. Ce monde social est un monde intersubjectif, un monde d'habitudes, dans lequel les actes de la vie quotidienne sont pour la plupart accomplis machinalement, dans lequel la réalité 
semble naturelle et sans problèmes. Cependant, l'expérience subjective d'un individu est inaccessible à un autre individu. En principe, cela devrait empêcher toute possibilité d'une réelle connaissance intersubjective. Ce n'est pourtant pas le cas, grâce à deux "idéalisations" utilisées par les acteurs :

- celle de l'interchangeabilité des points de vue d'une part: on peut échanger les places et avoir ainsi le même angle de vue que celui occupé précédemment par l'autre;

- celle de la conformité du système de pertinence d'autre part: tous les témoins d'un même spectacle supposent que les autres sont venus le voir pour les mêmes raisons que lui, qu'ils y portent tous un intérêt empirique identique, cela malgré leurs différences biographiques.

Considérées ensemble, ces deux idéalisations composent "la thèse générale de la réciprocité des perspectives", qui marque le caractère social de la structure du "monde de la vie" de chacun. [...]

Notre perception d'un objet est toujours le produit d'un travail de reconstruction qui prend en compte les "sédiments" de perceptions passées. C'est la raison pour laquelle le monde a pour nous un "horizon de familiarité" qui nous est fourni par le stock de connaissances dont nous disposons. Chacun dans le monde possède ainsi son "stock de connaissances disponibles", qui comprend aussi bien les croyances, les attentes individuelles que les règlements et les normes sociales. Ce stock repose sur des types expérientiels; il est fait de nos habitudes, c'est-à-dire de nos connaissances que nous pouvons mobiliser à chaque instant dans un grand nombre de situations sociales sans que nous ayons à faire d'efforts pour cela. Ce stock, qui s'enrichit constamment de situations nouvelles, nous permet d'interpréter le monde, interprétation sans laquelle toute action, ainsi que toute communication avec autrui, s'avérerait impossible. Les types permettent la transcendance de l'immédiateté, nous faisant considérer des objets semblables comme étant par essence les mêmes : la typification nous attache irrémédiablement au passé d'une part et implique l'anticipation d'expériences similaires d'autre part » (Coulon, 1994, p. 456).

L'approche de Schütz n'est ni cognitiviste (elle s'attache à une typicalité déjà là) ni ontologiste (elle vise les opérations de typifications réalisées par un sujet) (Cefaï, 1994). La réflexion opère en se rapportant à des types déjà disponibles, organisés en schèmes d'expérience, qui se sont articulés lors d'expériences passées et sont consignés dans les réserves d'expérience de l'acteur.

À la suite de François-André Isambert, on insistera surtout sur ce que la démarche de Schütz doit à la phénoménologie Husserlienne, particulièrement dans l'enracinement du type dans l'expérience anté-prédicative :

«Husserl part de l'idée d'une pré-organisation de notre expérience des choses. Cette pré-organisation repose sur la familiarité que nous avons avec ces choses perçues, par suite de notre expérience antérieure. Familiarité antérieure à tout jugement formel, qui commande une double attitude de notre part, traçant les horizons de l'objet perçu. L'horizon interne nous porte vers le semblable, donc vers "une infinité d'univers singuliers" (Husserl, 1970, p. 398) de la même famille, pourrait-on dire, que l'objet en question. L'horizon externe évoque les expériences possibles et l'exemple du chien, avec "sa manière typique de manger, de courir, de sauter, etc." est celui-là même qui sera repris par Schütz pour illustrer l'idée de type (Husserl, 1970, p. 402; Schütz, 1987, p. 13-14 et 79-80). Ainsi la typicité, au niveau de l'expérience la plus immédiate, se caractérise par cette familiarité qui fait de l'objet présent un exemplaire d'un type plus général et l'amorce d'un série d'expériences attendues. Sur cette base empirique peuvent être construits des concepts à caractère général. La notion de typicité s'élargit, selon Schütz lui-même, dans son dernier ouvrage. D'une part, la typicité de l'expérience est fonction de l'intérêt que nous portons à l'objet perçu. Schütz parlera de "pertinence" 
(relevance) des caractères de l'objet par rapport au genre d'attention que nous lui portons, et ce dans un sens plus pragmatique que celui auquel semblait penser Husserl (Husserl, p.1976, p. 30 Schütz, 1987, p. 14 et 104-106). Mais aussi l'expérience immédiate n'est pas celle d'une collection de choses figées et sans lien entre elles. Au contraire, les choses nous sont données comme altérables, variables, ce qui confère un certain flou à leur aperception, mais en même temps se trouve la certitude que cette variabilité est définie par des limites et des manières de varier particulières. " (Isambert, 1989, p. 308-309) sensibles à la générativité sociale des catégories (Quéré, 1994, p. 32-35). Ainsi, pour Garfinkel, les catégories sont à la fois des principes de construction de la réalité et des réalités objectives émergentes. Ceci ressort tout particulièrement dans son analyse du cas d'Agnes, une transsexuelle américaine. La réalisation des catégories est, dans cette perspective, orientée et contrainte par un schème de naturalité et de normalité, la «femme naturelle et normale». Ce schème fournit les règles et les méthodes de configuration des comportements, dispositions et attitudes reconnaissables comme réalisations de la catégorie «femme naturelle et normale ». Il consiste en un système de croyances conventionnelles: les gens souscrivent à des définitions tenues pour allant de soi, car légitimes, prenant généralement la forme d'attentes d'arrière-plan de la vie courante qu'ils utilisent comme repères pour configurer leurs comportements et pour constituer, par interprétation, ce qui leur apparaît en «apparences d'événements familiers». Sacks, pour sa part, a développé quelques intuitions essentielles pour explorer la valeur opératoire des catégories dans l'organisation séquentielle des cours d'action et dans la construction de l'observabilité, l'analysabilité et la descriptibilité des phénomènes sociaux (id., p. 35-37). Ces intuitions peuvent se résumer en quatre points : le caractère réglé de la sélection des catégories dans l'identification des personnes, des objets ou des événements et la nature normative des catégories (collections de droits et devoirs) ; le rôle de support joué par les catégories dans l'organisation et l'actualisation du savoir de sens commun sur le monde social ; le fait que l'organisation séquentielle et temporelle des cours d'action - la sélection d'une (paire de) catégorie(s) - pour définir une situation rende disponibles certains parcours à effectuer pour traiter cette situation, tandis que la co-sélection de catégories appariées sur le mode de relations standardisées assure la congruence des ordres de pertinence nécessaire à l'intercompréhension et à la coordination de l'action; enfin, la capacité à rendre les phénomènes visibles et observables.

manière plus spécifique, on peut observer une procédure consistant à attribuer un savoir particulier à une catégorie donnée ou à une série limitée de catégories, qui permet de considérer qu'un savoir est, d'un point de vue de sens commun, « détenu » par les membres de ces catégories, avec les droits et devoirs qui en découlent :

«Ce savoir basé sur la catégorie, ou même lié à la catégorie, peut se manifester comme tel en étant codé dans un argot propre à la catégorie. À l'inverse, si les membres visibles d'autres catégories s'attribuent ce savoir ou essaient de l'afficher, ils risquent d'être accusés de manquer de crédibilité, d'être des imposteurs, d'avoir "usurpé" ce savoir ou de se l'être arrogé abusivement, et ainsi de suite. » (Watson, 1995, p. 202)

Ainsi, la catégorie d'«outsider» se présente-t-elle comme support d'imputation de savoir défini sur une échelle continue d'anonymat et d'intimité. Tout ceci implique dès lors

Égypte/Monde arabe, 34 | 1998 
" une sociologie de la connaissance qui aborde les pratiques des gens, leurs typifications/catégorisations dans l'action, leurs activités et leurs interactions, leurs interactions communicatives prises dans le sens le plus large du terme. Nous avons besoin d'une sociologie de la connaissance qui soit véritablement praxéologique et puisse rendre compte empiriquement, et en détail, de la conduite des activités quotidiennes, de la vie quotidienne. » (id., p. 211)

\section{Confusion sémantique et différenciation pragmatique} donc par un jeu de typifications qui ne la distingue pas radicalement de l'activité du profane. Ceci est d'autant plus vrai que la typification ne consiste pas en une simple opération prédicative rapportant une situation à un paradigme, mais qu'elle opère de manière antéprédicative, en deçà donc de toute attribution intentionnelle de caractéristiques spécifiques à l'objet du jugement. Les types ne sont dès lors pas, a priori aumoins, des modèles servant de base à la comparaison, mais bien d'abord des structures de pertinence, l'« "index" pointant un problème » (Schütz, 1966, p. 128, cité par Quéré, 1994, p. 28).

Il reste toutefois que, si les typifications propres aux jugements professionnel et profane ne se distinguent pas par leur appui sur des stocks d'images différenciées, elles n'en aboutissent pas moins à des résultats différents. Je serais tenté d'expliquer cela par la double insertion pragmatique de ces jugements : la pragmatique biographique des acteurs et la pragmatique structurelle de leur activité. Si donc on ne peut en conclure à la spécificité sémantique des typifications ordinaire et judiciaire, il faut par contre s'interroger sur leur spécification pragmatique.

C'est au croisement d'expériences accumulées que se situent les typifications de l'acteur, expériences qui procèdent toutes d'une biographie qui, pour ce qui nous occupe, est partiellement professionnelle, partiellement profane. Pour reprendre l'expression de Schapp (1992), l'acteur est, en ce sens, empêtré dans ses histoires, c'està-dire dans tout ce qui est constitutif de sa biographie (en ce compris les interférences biographiques d'autrui). L'acteur connait du monde extérieur ces choses dont la « réputation» a traversé son histoire propre. Ces choses sont aussi bien des êtres que des objets dont il a fait l'expérience anté-prédicativement ou qu'il a évalués prédicativement (Dulong, 1994). Ainsi, la « réputation de la loi » est constituée de ces références au droit «officiel» qui interviennent dans des interactions ordinaires et exercent un effet pragmatique sur l'énonciation et l'action (Dulong, 1991). Confronté à une situation - p. ex. juger quelqu'un -, l'acteur se saisit d'une réputation, elle-même à la croisée d'histoires multiples dont il a fait l'expérience ou le jugement, pour éventuellement la rectifier (Dulong, 1994). L'extrait suivant du «Rapport d'instruction » (interrogatoires) sur l'affaire de la "jeune fille de Ma'âdî » devrait permettre d'illustrer le caractère préconstitué et stéréotypé de la démarche suivie par le magistrat.

Profitant de la présence des prévenus, détenus, à l'extérieur de la pièce où est menée l'enquête, nous les y avons appelés et leur avons demandé de répondre des accusations qui leur sont faites après les avoir informés que le Parquet ouvrait une procédure d'enquête contre eux. Ils ont tous admis (avoir pris connaissance de la chose) et nous leur avons demandé s'ils avaient un représentant devant comparaître avec eux pour la procédure d'enquête. Ils ont répondu par la négative. Nous avons fait sortir tous les prévenus de la pièce, à l'exception du premier. À 
l'examen, il s'agit d'un jeune homme d'une vingtaine d'années, d'environ 1,70 m, de corpulence moyenne, de teint foncé, portant un costume bleu avec des carreaux dans le bas et un pull-over bleu. Nous avons entrepris de le questionner en détail et il a répondu :

$\mathrm{R}$ : Anwar Ismâ'îl, 19 ans, balayeur d'entrepôts, résidant 'Izbat ( ?)

$\mathrm{Q}$ : Quels sont les détails de ce que tu reconnais?

[...] [suit l'interrogatoire complet]

$\mathrm{Q}$ : Tu es inculpé de participation avec d'autres à enlèvement et viol par contrainte.

Qu'as-tu à dire?

$\mathrm{R}$ : J'ai dit ce qui est arrivé.

$\mathrm{Q}: T u$ es également inculpé de participation avec d'autres à vol avec contrainte de ce qui a été susmentionné. Qu'as-tu à dire?

$\mathrm{R}: \mathrm{J}$ 'ai dit ce qui est arrivé.

$\mathrm{Q}$ : Tu es également inculpé de participation avec d'autres à enlèvement et séquestration illégale. Qu'as-tu à dire?

$\mathrm{R}$ : Oui. Ça s'est passé.

$\mathrm{Q}:$ As-tu des antécédents?

$\mathrm{R}:$ Non.

$\mathrm{Q}:$ As-tu autre chose à dire?

$\mathrm{R}$ : Non. Fin des propos de l'inculpé Anwar.

Nous avons ensuite mis l'inculpé en question sur le côté et nous avons appelé le second dans la pièce où est menée l'enquête et, à l'examen, nous avons rencontré un jeune homme d'une vingtaine d'années, d'à peu près 1,70 mètre, cheveux noirs courts, teint doré, portant un sweater jaune à l'origine, un pantalon citron et des chaussures noires. Nous avons ensuite entrepris de l'interroger en détail sur ce qui suit $[\ldots]$

C'est donc le caractère préétabli de la procédure qui me semble fondamental, non la différence de nature entre pensées profane et professionnelle. Par rapport à clémence et Doise qui affirment, bien que de manière nuancée, que justice et droit ne sont pas pensés de la même façon chez les profanes et les professionnels et qu'il est attesté que la logique professionnelle est de nature plutôt analytique, là où la logique profane est davantage représentationnelle (Clémence et Doise, 1995, p. 376), cette étude tend ainsi à montrer qu'il ne faudrait pas opposer trop radicalement les deux sphères de pensée. Le savoir profane se fonde le plus souvent sur des éléments de savoir technique et, inversement, le savoir professionnel comporte un substrat profane important. En fait, il importe surtout de constater à quel point les éléments sont imbriqués, chacun des deux types de sens de la justice s'appuyant sur des bribes de savoirs partagés. La différence essentielle vient peut-être alors plus des modes d'énonciation des règles de référence que de la substance même de ces règles, de la pragmatique de leur énonciation et non de leur sémantique. Bernard Jackson montre en tout cas parfaitement qu'aussi bien les faits, la règle, le jugement et la doctrine sont l'objet d'une appréhension narrative (une appréciation par rapport à des modèles) (Jackson, 1988 et 1995).

On constatera donc logiquement la nature doublement (à la fois professionnelle et profane) réflexive des typifications du droit et de la justice. Insérées dans un jeu d'histoires multiples, empêtrées dans des complexes de "réputations ", à l'intersection de la réceptivité antéprédicative et du jugement prédicatif, les typifications se constituent et se modifient constamment de par leur propre dynamique, configurent le contexte et reçoivent un complément de détermination par lui. On passe « de l'histoire de quelqu'un, avec sa complexion singulière d'horizons, à un récit de cas qui puisse faire jurisprudence, constituer une règle» (Dulong, 1994, p. 292). Ainsi en va-t-il du travail du juge, par exemple, qui d'un suspect fera un prototype, lequel prototype ne 
pourra pas ne pas influer sur l'appréhension du suspect, ou qui d'une affaire fera une cause, laquelle cause transformera le traitement de l'affaire.

Les différences entre professionnel et profane se situent donc ailleurs, dans la mise en scène de l'activité professionnelle (cf. Goffman), dans la narrativisation de la pragmatique professionnelle (cf. Jackson), dans l'organisation de l'activité pratique (cf. Sacks). Je laisserai de côté l'approche goffmanienne pour n'aborder que les deux autres.

D'un point de vue sémiotique, la notion de "professionnel » est un attribut (Jackson, 1994). Le syntagme narratif et les typifications narratives peuvent ainsi s'appliquer, non seulement au contenu d'un récit ou d'une action, mais aussi à l'acte même de communication de ce récit ou de cette action. Utilisant la métaphore de l'arbitre de football, Jackson montre que le professionnalisme est véhiculé par le transfert de certaines modalités à l'intérieur de la pragmatique narrativisée de l'activité de juger plutôt que par une application déductive par le juge des règles du jeu aux faits qu'il rencontre sur un terrain quelconque. Ce transfert s'opère doublement : par la volonté d'«agir comme un bon juge»; par la communication du fait que l'obligation est apparemment satisfaite. Il s'agit donc du transfert, non pas seulement d'un savoir, mais aussi et surtout d'un savoir-faire, le professionnalisme devenant ainsi le moyen par lequel un client est persuadé que le savoir-faire qui lui est transmis est "compétent " (transfert d'une valeur sémiotique). Le propre du sens professionnel du droit ne tient donc pas tant à des différences dans les typifications du droit qu'à la modalisation de l'activité juridique. Adopter un cadre d'analyse faisant une part à la narrativisation de la pragmatique permet de restituer au comportement des participants à l'interaction juridique - relations client-avocat, jugement, cours, etc. - toute son importance (Jackson, 1988, p. 88), que ce soit dans la construction des faits ou dans la construction et l'application du droit. Ceci vaut en toutes circonstances, que les questions ne soient juridiquement pas controversées (comment la conviction du fait que ce n'est pas controversé a-t-elle été acquise ? qu'est-ce que « penser comme un juriste »? quelle est la part du non institutionnel dans l'évaluation ?) ou que l'affaire porte exclusivement sur un point de droit - ce sont alors des processus de perception, de stockage, de restitution, d'énonciation des faits et du droit qui sont engagés (cf., p. 112-129).

Les sens ordinaire et judiciaire du droit et de la justice procèdent toutefois et avant tout d'activités pratiques. Les typifications dont ils font l'objet ne sont sans doute pas tant des opérations prédicatives que des "méthodes pour des activités pratiques», des «formules d'opérations possibles» (Quéré, 1994, p. 37). C'est alors à l'organisation de ces activités pratiques qu'il faut d'abord s'intéresser. En affirmant le primat du pragmatique sur le sémantique, nous rejoignons la préoccupation de Sacks pour l'ordre social et la façon dont on peut en rendre compte, dans la mesure où il s'agit d'une affaire familière et reconnaissable. L'activité ordinaire et judiciaire peut alors être considérée comme une activité sociale méthodique, procédurale, qui ne peut être envisagée que dans une perspective contextuelle ne voyant pas dans les catégories des dépôts de significations indépendants des circonstances de leur emploi, mais bien des ressources publiques et partagées utilisées pour donner un sens socialement standardisé aux situations (Watson, 1994, p. 152-154). Ainsi, les catégories auxquelles correspond la paire relationnelle standardisée "professionnel/profane» (les deux catégories sont conçues comme "allant ensemble ») sont-elles le lieu d'un ensemble de droits et d'obligations dont les éléments sont distribués selon un ordre lié 
intrinsèquement au contexte d'avant-plan, l'activité juridique, et extrinsèquement au contexte contingent d'arrière-plan.

L'interrogatoire des délinquants mené par le Procureur permet d'illustrer cette question de la différenciation pragmatique. On peut d'abord observer un mélange de règles de procédure et de mécanismes conversationnels quotidiens, ce qui signifie que les environnements institutionnel et interactionnel de l'action opèrent simultanément. Alors que, dans la vie quotidienne, il existe de fortes contraintes sociales mettant un frein à la formulation d'accusations, il $\mathrm{y}$ a, dans le contexte judiciaire, de simples contraintes procédurales encadrant l'énoncé des accusations (Komter, 1994). Le document intitulé «Inventaire des éléments de preuve, affaire criminelle 276/1985, Ma'âdî, enregistrée sous le $n^{\circ} 32 / 1985$, Tribunal plénier du sud du Caire ", donne l'illustration de la narration d'une accusation insérée dans une structure formelle la rendant pertinente pour l'imputation juridique de conséquences pénales.

Au nom du Dieu Clément Miséricordieux

Parquet du sud du Caire

Mlle..., âgée de 17 ans, étudiante à la faculté d'éduction musicale, domiciliée au 95 A, av. 'Abd al-'Azîz Al Sa'ûd, arrondissement de Misr al-Qadîma, témoigne du fait que, le 17 janvier 1985, elle était en compagnie de son fiancé, Ahmad Hamdî Hasan Imam, et qu'alors qu'ils étaient arrêtés en voiture, rue du Canal à Ma'âdî, l'accusé Salâh Shawqî 'Ait Abu Halâwa les a menacés en exhibant un couteau (corne de gazelle) et a ordonné à son fiancé de descendre de la voiture, [suit le témoignage de la victime] [...]

M..., âgé de 24 ans, directeur d'une société de produits textiles, domicilié au $95 \mathrm{~A}$, av. 'Abd al-'Azîz Al Sa'ûd à Manyal, arrondissement du commissariat de Misr alQadîma, témoigne du contenu de ce dont a témoigné le premier témoin (femme) et ajoute que, pendant sa détention par les accusés sur la voie publique, le premier accusé s'est emparé d'un bracelet aux fermoirs en or, de même que le deuxième accusé s'est emparé de sa montre quand ils se sont dirigés avec lui vers le taxi, ceci sous la menace pour chacun des deux accusés d'un couteau et en présence du reste des accusés, à l'exception du sixième. Il ajoute que le premier accusé l'a frappé avec le couteau au moment de l'emmener lui et la victime (femme) à l'intérieur du taxi que conduisait le cinquième accusé.

Observations

1. Le premier accusé a rapporté [...] qu'il avait convenu avec les deuxième, troisième, quatrième et cinquième accusés d'enlever n'importe quelle femme qu'ils rencontreraient et de la violer [...].

2. Le deuxième accusé a rapporté dans le procès-verbal d'arrestation la même chose qu'avait rapportée le premier accusé dans le procès-verbal d'arrestation. Il a reconnu dans le procès-verbal de renouvellement de sa détention, en date du 21 janvier 1985, et dans l'enquête du Parquet que les premier, troisième, quatrième et cinquième accusés avaient volé les deux victimes par la contrainte, qu'il avait volé la montre de là victime (homme) sur la voie publique en le menaçant d'utiliser le couteau qu'il portait et qu'il avait embrassé et étreint la victime (femme).

[...]

6. Le sixième accusé a reconnu dans le procès-verbal de renouvellement de sa détention, en date du 21 janvier 1985, qu'il savait que la victime (femme) avait été enlevée et qu'il a reçu les deux bagues et la chaîne en or en retour de la mise à disposition de l'endroit où les accusés ont violé la victime (femme).

[...]

On peut penser que les contraintes sociales freinant la formulation d'une accusation sont en quelque sorte neutralisées par l'officialité d'une fonction, celle de procureur 
pour le cas qui nous concerne, qui institutionnalise et ritualise la construction et la présentation de l'accusation.

Le récit du délinquant est également construit par le Procureur, de telle sorte qu'il anticipe les usages que le tribunal pourra en faire et qu'il devance les défenses possibles du délinquant. Komter parle d'une forme d'hyper-accusation englobant les positions de transaction que le défenseur pourrait adopter de même que l'admission sélective qu'il pourrait opérer de ce qui lui est imputé (id.). Cette anticipation est manifeste dans l'inventaire des éléments de preuve.

Mlle..., âgée de 17 ans, étudiante à la faculté d'éduction musicale, domiciliée au 95 A, av. 'Abd al-'Azîz Al Sa'ûd, arrondissement de Misr al-Qadîma, témoigne du fait que, le 17 janvier 1985, elle était en compagnie de son fiancé, Ahmad Hamdî Hasan Imâm, et qu'alors qu'ils étaient arrêtés en voiture, rue du Canal à Ma'âdî, l'accusé Salâh Shawqî 'Alî Abu Halâwa les a menacés en exhibant un couteau (corne de gazelle) et a ordonné à son fiancé de descendre de la voiture. Quand il a refusé, l'accusé a crevé la roue avant-droite de la voiture et, quand le premier a fait tourner le moteur pour s'en aller, il a crevé la roue arrière-droite en utilisant le couteau précité. En dépit de cela, la victime (homme) a pu conduire la voiture sur une courte distance; il lui fut ensuite impossible de poursuivre sa route en raison du dommage subi aux deux roues. Il s'est alors arrêté pour remplacer l'une des deux roues. Il avait entrepris de le faire quand il fut surpris une nouvelle fois par l'accusé qui demanda à sa fiancée une somme de cinquante livres en la menaçant du couteau. La victime (homme) sortit alors son porte-monnaie et lui donna ce qu'il contenait, c'est-à-dire un montant de vingt-cinq livres. Il l'a ensuite menacé d'enlever la victime (femme). Il lui a fait comprendre qu'elle était sa fiancée. Il a toutefois poursuivi et s'est installé sur le siège arrière de leur voiture. Il lui ordonné de la mettre en route et il s'est conformé à cet ordre. Il l'a ensuite contraint à s'arrêter après une courte distance près d'un taxi transportant les accusés, du deuxième au cinquième. Ils en sont descendus, le deuxième tenant un couteau (corne de gazelle). Ils les ont obligés à monter dans le taxi conduit par le cinquième accusé et ils sont partis pour un autre endroit sur la voie publique où les accusés sont sortis, à l'exception du premier accusé, pour conduire la victime (homme) en un lieu un peu éloigné de la voiture. Le premier accusé, qui était resté sur le siège arrière de la voiture, a entrepris de lui ôter ses vêtements de force en la menaçant du couteau et s'est allongé sur elle. Il n'a pu faire pénétrer une partie de son membre viril dans son vagin qu'avec peine. À sa suite, ce fut le deuxième accusé qui s'est précipité sur elle dans la voiture et s'est allongé sur elle, quand retentirent des coups de feu. Lui et les autres accusés se sont empressés de monter dans la voiture, la victime (homme) avec eux, et ils sont partis pour la région des tombes à la recherche d'un abri pour poursuivre leurs actes sexuels. Ils n'y sont pas parvenus; ils se sont alors dirigés vers une zone habitée et se sont arrêtés devant un bâtiment au pied duquel il y avait un garage vide de voiture. Le premier accusé est descendu et a rencontré le sixième accusé; et ils se sont rendus ensemble vers le garage. Le premier accusé est ensuite revenu à la voiture et a demandé à la victime (femme) de rentrer dans le garage. Elle a obtempéré à l'ordre, ceci sous le regard du sixième accusé. Le premier accusé a ensuite sorti une couverture et un coussin et les a posés à l'intérieur d'une pièce attenante au garage dans laquelle les accusés, à l'exception du sixième, ont entrepris de rentrer. Chacun a ensuite entrepris de se dévêtir et de s'allonger sur elle en essayant de faire pénétrer son pénis dans son vagin. Mais elle s'est efforcée de leur résister et elle a été blessée à la main gauche du fait de sa résistance. Elle a ajouté que le premier accusé, quand il l'a conduite dans la pièce attenante au garage, s'est emparé de deux bagues qu'elle portait.

[...]

Observations 
1. Le premier accusé a rapporté [...] qu'il avait convenu avec les deuxième, troisième, quatrième et cinquième accusés d'enlever n'importe quelle femme qu'ils rencontreraient et de la violer. [...] Il a reconnu dans le procès-verbal du renouvellement de sa détention, en date du 21 janvier 1985, la même chose qu'il avait rapportée dans le procès-verbal d'arrestation.

2. Le deuxième accusé a rapporté dans le procès-verbal d'arrestation la même chose qu'avait rapportée le premier accusé dans le procès-verbal d'arrestation. Il a reconnu dans le procès-verbal de renouvellement de sa détention, en date du 21 janvier 1985, et dans l'enquête du Parquet que les premier, troisième, quatrième et cinquième accusés avaient volé les deux victimes par la contrainte, qu'il avait volé la montre de la victime (homme) sur la voie publique en le menaçant d'utiliser le couteau qu'il portait et qu'il avait embrassé et étreint la victime (femme).

3. Le troisième accusé a reconnu dans l'enquête du Parquet la même chose que celle rapportée par le premier accusé et il a ajouté qu'il avait saisi de force la victime (femme), s'était allongé sur elle et l'avait embrassée.

4. Le quatrième accusé a reconnu dans l'enquête du Parquet la même chose que celle rapportée par le premier accusé et il a ajouté qu'il avait saisi la victime (femme), l'avait étreinte et l'avait embrassée, de même qu'il a reconnu dans le procès-verbal de renouvellement de sa détention, en date du 21 janvier 1985, avoir participé au viol de la victime (femme), l'avoir embrassée et lui avoir mordillé les seins.

5. Le cinquième accusé a reconnu dans le procès-verbal du Parquet la même chose que celle rapportée par le premier accusé et il a ajouté qu'il avait saisi la victime (femme), l'avait étreinte et l'avait embrassée, de même qu'il a reconnu dans le procès-verbal de renouvellement de sa détention, en date du 21 janvier 1985, qu'il avait convenu avec les quatre premiers accusés d'enlever la victime (femme), de la violer et de voler ce qu'elle possédait par la contrainte.

6. Le sixième accusé a reconnu dans le procès-verbal de renouvellement de sa détention, en date du 21 janvier 1985, qu'il savait que la victime (femme) avait été enlevée et qu'il a reçu les deux bagues et la chaîne en or en retour de la mise à disposition de l'endroit où les accusés ont violé la victime (femme).

[...]

40 Comme on le faisait remarquer précédemment, l'intentionnalité des agresseurs est rendue manifeste, ce qui permet d'anticiper toute velléité de défense par l'idée d'une action impulsive. La majorité d'un des prévenus est également établie, ce qui l'empêche d'alléguer de son âge pour échapper à la sanction prévue par la loi.

[...] 12. Il ressort du rapport médical initial que l'accusé Ashraf Hasan Jamîl a plus de 18 ans. [...]

Le défenseur dispose de différents types de stratégie lui permettant d'échapper à sa responsabilité ou de l'atténuer. Il peut, d'abord, fournir des descriptions alternatives. La déposition d'un des agresseurs peut ainsi insister sur le rôle prépondérant d'un autre agresseur dans le crime qui a été commis, alors même que la déposition de ce dernier tend à davantage souligner le caractère collectif du crime.

\section{Anwar Isma'îl}

$\mathrm{Q}$ : Êtes-vous tombés d'accord pour prendre n'importe quelle femme sur la route?

$\mathrm{R}$ : Nous avons été d'accord pour enlever une femme et que Salâh nous la prenne.

Salâh Shawqî

$\mathrm{Q}$ : La conversation s'est-elle engagée entre vous sur le terrain de la recherche d'une femme?

$\mathrm{R}$ : Oui, nous sommes tombés d'accord pour chercher une femme.

42 Une autre possibilité consiste à donner des motifs à l'action, des accounts dans le vocabulaire ethnométhodologique. Nous pouvons les appeler techniques de neutralisation. Dans notre affaire, le Procureur ne semble pas avoir laissé une place 
importante à l'usage de pareilles techniques qui pouvaient mener à l'atténuation du crime et, en conséquence, de l'accusation. Ceci étant, il faut relever l'argument d'un des agresseurs qui affirme avoir proposé au fiancé de la victime que la fille soit leur bien partagé (shirka), ce que le garçon aurait accepté. On peut penser que cet élément visait à atténuer la culpabilité des agresseurs en présentant l'affaire sous un jour de quasiconsentement des victimes.

Salâh Shawqî

Je l'ai aidé à changer la roue et après en avoir fini, il est monté dans la voiture. Je me suis approché en tenant le couteau et je lui ai dit: la fille qui est avec toi, c'est un bien partagé (shirka). Me voyant avec mon couteau, il a été d'accord. Je lui ai dit : monte devant, et nous sommes allés un peu plus loin [...].

De même en va-t-il de cet autre motif avancé par un agresseur, qui tend à expliquer la décision de lancer une " chasse à la femme " par la déception qu'un autre des prévenus a connue quand il a vu la fille avec laquelle il flirtait partir avec un autre homme.
Anwar Ismâ'îl
[...] Nous avons rencontré Salâh Abu Halâwa, qui connaît Ashraf, et il a dit qu'il se trouvait avec une femme et qu'un indic (de police) la lui avait prise et lui avait pris cinq livres. Il est monté avec nous et a dit, alors que nous avancions un peu : je vais vous enlever n'importe quelle femme. [...]

De manière générale, le défenseur semble répondre aux questions, non seulement en fonction de leur contenu factuel, mais aussi en fonction de ce qu'elles impliquent comme condamnation morale (blame-implicative nature). Ainsi, par exemple, en va-t-il de ce défenseur qui admet être rentré dans la pièce pour y violer la fille, mais qui explique que, parce que la fille lui a rappelé le caractère mauvais de son action, il a quitté la pièce sans avoir eu de relations avec elle.

\section{Anwar Ismấîl}

$\mathrm{Q}$ : Quels sont les faits sexuels que tu as commis sur la victime (femme)?

$\mathrm{R}$ : Quand je suis rentré, je l'ai trouvée appuyée sur le coussin. Je me suis apprêté à coucher avec elle, je l'ai embrassée, je l'ai prise à la taille et je l'ai étreinte. Quand elle m'a dit : harâm 'alayk, je l'ai laissée et je suis sorti.

Le défenseur doit aussi résoudre le dilemme consistant à fournir des informations sans porter atteinte à ses intérêts, à montrer sa volonté de coopérer tout en restant sur la défensive. Une admission partielle le rend coopératif, par exemple, tandis qu'une dénégation partielle lui permet de se défendre. Une autre stratégie de défense consiste, pour le défenseur, à estomper sa participation active aux événements. Il se fait ainsi, jusqu'à un certain point, disparaître comme agent de l'histoire. Les documents sur lesquels on a pu travailler laissent cependant apparaître une admission totale. L'explication tient, sans doute, au fait que l'enquête du Parquet fait suite à celle de la police. Il n'est pas rare, dans ce contexte, que les prévenus répètent un scénario construit par les inspecteurs de police et répété dans leurs locaux.

Si le travail du Procureur est officiellement d'examiner ce qui s'est objectivement passé et quelles sont en conséquence les responsabilités du défenseur, il se révèle également préoccupé de la culpabilité morale de ce dernier. Il n'est toutefois pas possible de relever, dans notre affaire, de formulation explicitement morale dans l'interrogatoire des prévenus par le Parquet. Ce type de propos doit probablement plus facilement apparaître à l'occasion des audiences des tribunaux et, plus particulièrement, au cours des interrogatoires et contre-interrogatoires de la Cour d'assises. Ceci dit, on notera qu'alors même qu'il recherche les faits, le Procureur dévoile souvent une position davantage morale. Il demande ainsi au défenseur, par exemple, d'évaluer ses actes. Ou 
il demande au défenseur de répéter ses aveux, ce qui lui permet de souligner les actes qu'il avoue et de lui faire admettre leur caractère mauvais. Ceci apparaît très clairement, dans l'affaire qui nous sert de support, à la lecture de l'interrogatoire du Parquet.

\section{Anwar Ismâ'îl}

$\mathrm{Q}:$ La fille se trouvait-elle dans cette situation de son plein gré ?

$\mathrm{R}:$ Non.

Q: La victime (femme) s'est-elle rendue avec vous dans le lieu où la victime (femme) a été agressée de son plein gré ?

$\mathrm{R}$ : Non. Nous les avons emmenés et les avons fait monter dans le taxi avec nous et nous leur avons dit : nous vous conduisons au poste.

On notera enfin que le défenseur, pour sa part, ne cherche généralement pas à contester la moralité qui est en jeu; il tente juste de diminuer ce que cela pourrait impliquer quant à sa responsabilité morale. Aussi bien le Procureur que le défenseur semblent attachés à restaurer l'ordre moral, le Procureur en faisant en sorte que la conformité morale du défenseur soit exprimée, le défenseur en confirmant la moralité dominante tout en évitant les conclusions corollaires qui pourraient être tirées sur sa propre moralité. Nous pouvons observer ici une espèce de consensualisme différencié : accord sur la moralité dominante sur laquelle le Procureur et le défenseur convergent, différencié quant à sa mise en scène concrète dans l'affaire qui les réunit.

\section{BIBLIOGRAPHIE}

\section{CASSIRER E. :}

- 1972, La philosophie des formes symboliques. I. Le langage, Paris, Minuit.

- 1977, Substance et fonction, Paris, Minuit.

CEFAÏ D., 1994, « Type, typicalité, typification. La perspective phénoménologique », dans B. Fradin, L. Quéré et J. Widmer (sous la dir.), L'enquête sur les catégories, Paris, Éd. de l'EHESS, coll. Raisons pratiques, vol. 5 .

CICOUREL A. V., 1979, La Sociologie cognitive, Paris, PUF.

CLEMENCE A. et DOISE W., 1995, « La représentation sociale de la justice : une approche des droits dans la pensée ordinaire ", L'Année sociologique, 45/2, p. 371-400.

COULON A :

- 1987, L'ethnométhodologie, Paris, PUF.

- 1994, « Alfred Schütz », dans Van Meter K. M. (sous la dir.), La Sociologie, Paris, Larousse.

DULONG R. :

- 1991, « "On n'a pas le droit... " Sur les formes d'appropriation du droit dans les interactions ordinaires ", dans Chazel F. et Commaille J. (sous la dir.), Normes juridiques et régulation sociale, Paris, LGDJ. 
- 1994, "Quand l'histoire disqualifie la catégorie. La perspective historiale de W. Schapp ", dans B. Fradin, L. Quéré et J. Widmer (sous la dir.), L'enquête sur les catégories, Paris, Éd. de l'EHESS, coll. Raisons pratiques, vol. 5 .

DUPRET B., 1998, « La typification des atteintes aux bonnes mœurs : approche praxéoiogique d'une affaire égyptienne ", International Journal for the Semiotics of Law, vol. 11, n 33, p. 303-322.

FERRIÉ J.-N :

- 1997a, « Solidarité sans consensus en Égypte : un cadre d'analyse », Les Annales de l'autre islam, 4.

- 1997b, Le Régime de la civilité. Public et réislamisation en Égypte, Mémoire pour l'habilitation à diriger des thèses de science politique, Paris, Institut d'Études Politiques.

FLETCHER G., 1978, Rethinking Criminal Law, Boston, Little, Brown \& Co.

GARFINKEL H., 1967, Studies in Ethnomethodology, Englewood Cliffs, NJ, Prentice Hall.

HUSSERL E. :

- 1970, Expérience et jugement, Paris, PUF.

- 1976, Idées directrices pour une phénoménologie, Paris, PUF.

ISAMBERT F.-A., 1989, « Alfred Schütz entre Weber et Husserl », Revue française de sociologie, 30/2, p. 299-319.

JACKSON B. S. :

- 1988, Law, Fact and Narrative Coherence, Liverpool, Deborah Charles Publications.

- 1994, " Towards a semiotic model of professional practice, with some narrative reflections on the criminal process ", International Journal of the Legal Profession, 1/1, p. 55-79.

- 1995, Making Sense in Law. Linguistic, Psychological and Semiotic Perspectives, Liverpool, Deborah Charles Publications.

KOMTER M., 1994, « Accusations and defences in courtroom interaction », Discourse and Society, $\mathrm{n}^{\circ} 5 / 2$, p. $165-187$.

QUÉRÉ L, 1994, « Présentation », dans B. Fradin, L. Quéré et J. Widmer (sous la dir.), L'enquête sur les catégories, Paris, Éd. de l'EHESS, coll. Raisons pratiques, vol. 5.

SCHÜTZ A. :

- 1966, Collected Papers III, La Haye, Martinus Nijhof.

- 1987, Le chercheur et le quotidien, Paris, Méridiens Klincksieck.

SHARROCK W., WATSON R., 1990, « L'unité du faire et du dire. L'action et l'organisation sociales comme phénomènes observables et descnptibles », dans P. Pharo et L. Quéré (sous la dir.), Les formes de l'action, Paris, Éd. de l'EHESS, coll. Raisons pratiques, vol. 1.

WATSON R., 1994, «Catégories, séquentialité et ordre social. Un nouveau regard sur l'œuvre de Sacks », dans B. Fradin, L. Quéré et J. Widmer (sous la dir.), L'enquête sur les catégories, Paris, Éd. de l'EHESS, coll. Raisons pratiques, vol. 5 .

\section{NOTES}

1. «Cet ensemble de procédures consiste en une détermination réciproque d'une structure et de détails, dans un mouvement de va-et-vient. Des apparences particulières, des "preuves 
documentaires" singulières, sont prises comme "indiquant" ou "représentant" une structure supposée sous-jacente. Cette structure est elle-même utilisée comme un outil d'interprétation qui, à son tour, renforce l'interprétation des preuves documentaires, considérées à la fois séparément et comme s'ajustant entre elles pour former un ensemble. » (Watson, 1995, p. 211)

INDEX

Mots-clés : ethnométhodologie, praxéologie, tribunal, éthique, espace public, moralité

AUTEUR

BAUDOUIN DUPRET

CNRS/CEDEJ 